\title{
Evaluación neurológica en pacientes con hipotiroidismo congénito diagnosticado por rastreo neonatal
}

\author{
Marcos Manriquez. O. '; Lilianette Nagel B. ${ }^{2}$ : Ximena Vivanco W. ${ }^{3}$
}

\begin{abstract}
Resumen
Ob,etwo. Desc bir el resultedo de la evaluación neurológicc de seguirtienlo en niños con hipotiroidismo congénito celec ados pcr rasliec neono ol y su correlación con variables que delerminan pronósico desíavorable para en desarollo cel sistemo nenioso central. Diseño: segumieno prospectivo. Método: controles periódicos de evainacióneurológ ca de 37 niz̄os con hipchroicismo congénito delectedo por rastreo de TSH en recién nacidos y confirmado por TSit. T4 y cirilgrafio tiroidea en el llamado de recuperación enle morzo de 1992 y morzo 1995 en Santiogo. Chile. Variable dependienle: axcren neurológico. Variables independientes: intensidad del hipotiroidismo según la concentración sérico inicial de T<, el resuliado de la cinligrofía y la edad en que comenzó el ratamierilo de sustitución de la homona liraidea. Se uthliź prueja de $\chi^{2}$. aceplándose como s.gnificalivas diferencias con $p<$

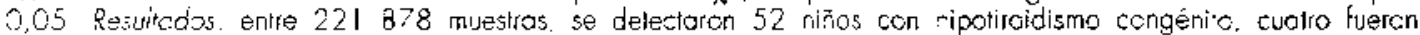
excluidos por sutrir. ademés, oras alecciores yje causabon caño revirológico y alros 11 por no cumplir rodos los contrile: de seguimiento. Enrre los 37 n:ños estudiados, nueve presenteron signos de déficit neurclógico en el curso del seguirriento. Se enccritraron asociaciones significarivas de eslos signos con la intensidad del hipotiroidismo pero no con lo cintigrafo o la edad de incio del tratamiento, que cas siempre fue precoz. Conciusiones: la relación entre gravedad del déricht hroideo al race y halazgos cnorrales en el seguimiento nejrológico sugieren efectos prenaroles de ducho célicil que es factor más relacionaco en le literatura con mol pronóstico.
\end{abstract}

(Palabras clave: ripotıcidismo congénito, sistemo nervicso.)

\section{Neurological evaluation in congenital bypothyroidism detected by neonatal screening}

Otiective to describe neurological find'ngs in infants with congenitcl hypolhyroidism cieteced by TSH scresnıng

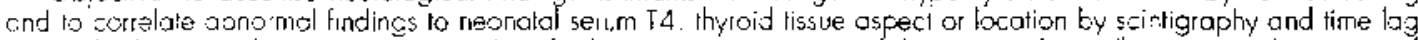
to initid sibstitution theropy. Foriens and method: prospertive one year folow up sludy to all patients with congenitol hypotriyroid on derected fom rarch 1992 thoughou: mart $: 905$ by neonotal 'S-1 screening at metropalitan Santicgo. Chile comf med by recall TSH, T4 < $10 \mu \mathrm{g} / \mathrm{dl}$ anc Tc $99 \mathrm{~m}$ thyroid scinligraphy. Dependent variable wos any neviologicol delicil. Independeril variubles were severity of congenilal hypothyroidism bosed on T4 seric in tiol levels, ontome asfecl anc bcation $0^{*}$ Ihycid tissue by scin igraphy, and lime in weeks trom tirth to iritial substitution therapy Resuits: 52 cases of congenita typothyroidism were detected among 221878 screened newborn infonss. fcur were 'ost to Sollower, 11 hod conditions other than hypothyroicism trat coulc explain any neurological abnomaling and 37 were available for evcluation. Nire susjects stowed some kind of nevrological deficit sometime along a che vear folow-up peiod. A jignificant asscciation was ound ameng severily of thyroid deficiency and atenomal neurregical find ngs. No relaronships were found with scint groohic fincigs or age at inithal therapy because this was always irstifuted betore age one mork. Conclusionstrelationship between severity of circuloring TA se- Jm jeticit and postnctal abrormal riesrologicol fincings may be eviderice of prenasal efects ol hypalhyroidism.

(Koy words: congenitr.l hypothyroidism, cenl al nervous system.)

1. Servicio de Neuropsiquiatria infantil. Hospital San Juall de Dín:

2. Prograina de Formación de Especialisa en Pediacria. Faculad de Medicina, Liniversidad de Chile. Departamenco de Pediatría y Cirugŕa Infantil Occidente.

3. Departamento de Pediatría y Cirugia Infantil OccidenIt. Facultad de Medicina. Universidad de Chile.
El hipotiroidismo congénito es la enfermedad endocrina más frecuente en la infancia, produce efectos devastadores sobre el crecimiento y desarrollo, pero es una de las pocas causas de retardo mental en que este puede ser prevenido. si se diagnostica y trata oportunamente. Su 
identificación clf́nica en los recién nacidos (RN) es difícil, pero desde 1975 se cuenta con medios efectivos de laboratorio de rastreo para su pesquisa precoz.

La glándula tiroides se origina en el piso de la faringe durante la cuarta semana de gestación. Desde la undécima semana es capaz de concentrar yodo y secretar tiroxina y a los cuatro y medio meses se ha activado el eje hipotalámico-hipofisiario-tiroideo. Esta hormona influye de manera importante en el desarrollo de] sistema nervioso desde los últimos meses de la vida fetal y a lo largo de los dos primeros años de vida, especialmente en los procesos de mielinización, arborización dendrítica, formación de sinapsis y migración neuronal ${ }^{1}$.

En la vida intrauterina la tiroxina es producida por el feto, si bien en la última semana podría existir paso de la hormona desde la madre. Parece ser que, en los casos más severos, el aporte materno no es suficiente $y$ los recién nacidos ya tendrían defectos en la maduración del sistema nerviosos que empeoraría a medida que se retarda el comienzo del tratamiento postnatal"1.

Desde 1992 se aplica en la Región Metropolitana un programa de rastreo neonatal mediante determinación de tirotropina (TSH) sérica, de acuerdo con las recomendaciones de la Organización Mundial de Salud ${ }^{2}$, en el cual se contemplan evaluaciones psicométricas y neurológicas periódicas de los niños detectados.

El objetivo de este estudio fue describir los hallazgos de la evaluación neurológica de seguimiento en niños detectados por el procedimiento y relacionarlos con la oportunidad del tratamiento ${ }^{1.2}$. la intensidad del hipotiroidismo ${ }^{3-6}$ y la atireosis $s^{6.7}$, factores que porencialmente afectan el pronóstico del desarrollo cognitivo y motor.

\section{Material y Método}

Se incluyeron Iodos los recién nacidos en que se detectó hipotiroidismo en la Región Metropolitana de Santiago entre el I de marzo de 1992 y el 31 de marzo de 1995. La detección se hizo midiendo TSH por método de ensayo radioinmunométrico (IRMA) en una muestra de sangre obtenida por punción de talón. cuyo procesauniento ha sido descrito anteriormente?

Se llamaron todos los recién nacidos cuya concentración de TSH en el examen de rastreo fuese igual o superior a $20 \mathrm{mU} / /$, para confirmar el resultado mediante ]RMA y medir T4 con radioinmunoensayo (RIA), en muesuras de suero, dando de alta a los niños en quienes TSH era menor de $10 \mathrm{mUL} / /$ y T4 mayor que $10 \mu \mathrm{g} / \mathrm{dl}$. Los niños con TSH igual o mayor a $10 \mathrm{mUI} / 1$ y T4 igual o menor a $10 \mu \mathrm{g} / \mathrm{dl}$, fueron calificados como hipotirojdismo congénito, se les realizó cittigrafía con Tc 99 e incorporó a un programa de seguimiento con contrales periódicos de psicometría y evaluación neurológica a los $2,4,6,8,10$ y 12 meses y. posteriormente, anual. Tan pronto se confirmó el diagnóstico, se indicó a los pacientes levotiroxina en dosis inicial de 10 a $15 \mu \mathrm{Hg} \cdot \mathrm{kg}$ - día, reduciéndola gradualmente hasta $506 \mu \mathrm{g} \cdot \mathrm{kg}$ - día a la edad de 12 meses. Todas las evaluaciones neurológicas fueron realizadas por el mismo examinador, quien no tenía información sobre la edad en que comenzó el tratamiento, la severidad y el tipo del hipotiroidismo. La informacion sobre el desartollo psicomotor se registró en una ficha esıándar empleando los criterios de Swaiman ${ }^{12}$, con márgenes de flexibilidad de seis semanas, La intensidad del hipotiroidismo fue estimada al confirmas el diagnóstico y considerada severa o grave si la concentración de T4 en el suero tomado en el llamado de confimación era igual o menor a $3,3 \mu \mathrm{g} / \mathrm{d} 13$ y menos severá si era mayor. El comienzo del tratamiento $0^{2,7}$ se consideró tardio después de un mes, pero, puesto que generalmente fue muy precoz, se agruparon arbitrariamence los casos en aquellos en que comenzó hasta en el día 21 de vida o posteriormente. El diagnóstico anatómico de atiria se hizo por cintigrafía.

La información se procesó en un programa EPI INFO 6. En el análisis estadístico se empleó la prueba de chi cuadrado $\left(\chi^{2}\right)$ y se aceptaron como significativas difetencias con probabilidad menor de 0.05 .

\section{Resultados}

En ese período se tomaron 221878 muestras, de las cuales 73 resultaron alteradas. En definitiva, 52 casos fueron considerados hipotiroidismo congénito, (res hipotiroidismo congénito tardío, seis hipotiroidismo congénito compensado y 12 hipertirotropinemia.

En este estudio sólo se consideraron los 52 pacientes con hipotiroidismo congénito detectado en el período neonatal, ya que las variables estudiadas son aplicables en sus casos, permitiendo comparaciones con otras experiencias publicadas. Cuatro de ellos fueron descartados por sufrir otras afecciones causantes de daño neurológico y 11 por no cumplir con el segujmiento neurologico establecido, siendo objeto de este informe los 37 pacientes restantes, 24 de los cuales eran mujeres.

En ntueve casos (24\%) la evaluación neurológica mostró alteraciones. En cuatro, estas consistian en síndrome piramidal (en un paciente el examen se había normalizado al año de edad); en los cinco restantes se registró retraso del de- 
sarrollo psicomotor de 2 a 3 meses, uno tenía. ađemás, hemiparcsia braquiocrural izquierda; en dos el desarrollo se había normalizado a la edad de un año.

En los nueve pacientes con alteraciones neurológicas la edad ósea estaba retrasada y en el período neonatal se habia detectado alguna de las características físicas comúnmente asociadas con hipotiroidismo (llanto ronco, ictericia, hernia umbilical, constipación, en[rianiento). Al cotejar las alteraciones neurológicas con la severidad del hipotiroidismo, siete $(46,7 \%)$ ocurriecron cntre los 16 niños con deficiencia severa y sólo dos $(9,1 \%)$ en los 22 casos menos severos, siendo significativa la relación entre ambas variables $\left(\chi^{2}-5,99 p<0,05\right)$.

La anomalía anatómica más frecuentemente registrada por cintigrafia ${ }^{6.7 .9}$ fue tiroides ectopica, que ocurtió en 18 casos, cinco de los cuales corresponden a los niños que mostraron alteraciones neurológicas $(27,8 \%)$. Estas últimas también ocurrieron en $3 / 8(37,5 \%)$ pacientes con atireosis y $1 / 8$ casos de bocio difuso. Los restantes tres pacientes, uno con glándula de baja captación pero ubicada normalmente y dos niños no evaluados con cintigrafía, no mostraron signos clínicos neurológicos. Estas relaciones no fueron significativas $\left(\chi^{2}=1,32 \mathrm{p}=\right.$ 0.5 !).

El examen ncurológico mostró alteraciones en $6 / 27$ pacientes en que el tratamiento de suslitución comenzo hasta la edad de 21 días y en $3 / 10$ de aquellos en que se inició más tarde, lo que no tiene significación estadística (Yates cotregido $\left.\chi^{2}=0,11 ; p=0,74\right)$.

\section{Comentario}

En la mayoría de las publicaciones de los últimos cinco años se muestran evaluaciones en el área cognitiva, con diferentes escalas ${ }^{2.5,8-11}$. El examen ncurológico resultó completamente normal en más de las tres cuartas partes de esta serie, todas las alteraciones detectadas eran más bien leves y tendían a normalizarse después de la edad de un año.

La relación estadísticamente significativa entre defectos neurológicos e intensidad del déficit tiroideo podria ser evidencia de hipotiroidismo in mero?. Este es el factor que se rekaciona con mal pronóstico en la literatura, sugirien- do efectos prenatales sobre el desarrollo neurológico, que el tratamiento precoz puede disminur sin evitar del todo.

La falta de asociación de las alteraciones neurologicas con las variables de momento de iniciación del tratamiento y ubicación anat6mica de la glándula tiroides probablemente se debe a que en la mayoría de los pacientes el tratamiento fue muy precoz.

Mediante el examen neurológico periódico y cuidadoso de estos pacientes durante la lactancia, parece posible detectar temprano a algunos que después pudiesen presentar problemas cognitivos y motores, permitiendo su manejo precoz y sistemático, por lo que puede ser conveniente seguirlos por plazos más largos.

\section{Agradecimientos}

Al Dr. Neison Vargas, por el analisis crítico de este malerial y sus sugerencias. Además debemos destacar que este tratajo no hubiera sido posible sin el aporte y experiencia desarrollada por el comite ministerial del programa nacional de hipotiroidismo congénito y fenilquetoлuria.

\section{Referencias}

I. Rakesh KG, Bathiet V. MD, Poptuni H, Gujrul R, NH. Brain metabolite changes on in vivo proton magnetic resonance spectroscopy in children with congenital hypothyroidism. J Pediatr 1995: 126: 389-392.

2. Abodorsky N, Vivanco $X$. Cuello $X$, et al.: Detección precoz de hıpotiroidismo congénito en Chile. Resultados en 24 meses. Rev Chil Pediatr 1995; 66: 140 . 144.

3. Konistra L. NMC; Lathe C. Msc; Vulsna T, NMP PhD. Schellekens I. Mh; Van der Meere, JPhd. Kalverbor, AfF $\mathrm{PhD}$ : Motor and cognitive development in children with congenital hypothyroidisın: A long-tenn evaluation of the effects of neonatal treatment. J Pediatr 1994: 124: 903-909.

4. Tillotson S, Fuggle PW, Smith I, Ades AE. Grant D: Relarion hetween bichemical severity and intelligence in early treated congenital hypothyroidism: a threshold effect. BMJ 1994; 309: 440-445.

5. Glorieux J, Lps. Dussauh J. NM Vin Vlient G. MD. Intellectual development at age 12 years of children with congenital hypothyroidism dingnosed by neonatal screening. J Pediatr 1992; 121: \$81-584

6. Simmons WF. Faggle PW. Grant DB. Smith J: Intellectual development at 10 years in early treated congenital hypothyroidism. Arch Dis Child 1994: 71 : 232.234

7. RochiccidlipR. Oxe B, Alexandre F. Tatiber MT: School athievement in children with, hypothyroidism 
detected at birth and search for predictive factors. Horm Res 199:: 39: 236-240.

8. Rovet $J F$, Eritich RM, Sorbara DL: Hypothyroidism: etiological and treatment factors affecting outcome. J Pediatr Psychol 1992; 17: 187-213

9. Glorietax J. Dussauth JH, MD. Morissetse, J. Desjardins M. Letarte J, MD. Guyda H, MD: Followup at 5 and 7 years of mental development in chijdren with hypothyroidism detected by Quebec Screening Program. J Pediatr 1985; 107: 913-915.
10. Department of Pedialrics. Darmouth Medical School, Lebanon, New Hampshire: Correlation of cognitive test socres and adequacy of treatment in adolescents with congenital hypothyroidism. J Pediatr 1994; 124: 38.3-387.

11. Migr $R$ : Mental development in congenital hypothyroidism after neonatal sereening. Arch Dis Child 1987; 62: 1050-1055.

12. Swaman-Ketheth $F$, MD: Pediatric neurology. principles and practice. $1994 ; 3 ; 43-52$.

Esta publicación está disponible en copias de microfilmes de 16 y $35 \mathrm{~mm}$ y microfichas de $105 \mathrm{~mm}$, las que pueden solicitarse a:

University Microfilms Internacional 300 North Zeeb Road Ann Arbor, Michigan 48106, USA.

This journal is also available in $16 \mathrm{~mm}$ microfilm, $35 \mathrm{~mm}$ microfilm and $105 \mathrm{~mm}$ microfilm copies through:

University Microfilms international, 300 North Zeeb Road, Ann Arbor, Michigan 48106, USA. 